\title{
Doença do refluxo \\ gastroesofágico no idoso
}

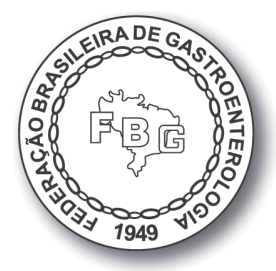

Ary Nasi

Natália Sousa Freitas Queiroz

\section{INTRODUÇÃO}

Considerando-se que a proporção de idosos na população vem aumentando expressivamente nas últimas décadas, é bastante pertinente a ideia de rever particularidades das afecções digestivas nessa faixa etária. A doença do refluxo gastresofágico (DRGE), considerada uma das afecções digestivas de maior prevalência nos países ocidentais ${ }^{1}$, apresenta particularidades no idoso. $\mathrm{O}$ objetivo deste capítulo é apresentar uma revisão sobre o tema, contribuindo para o diagnóstico e o tratamento da doença em idosos.

\section{DEFINIÇÃO}

O II Consenso Brasileiro sobre Doença do Refluxo Gastroesofágico² define a doença como: afecção crônica decorrente do fluxo retrógrado de parte do conteúdo gastroduodenal para o esôfago e/ou órgãos adjacentes ao ele, acarretando variável espectro de sintomas e/ou sinais esofágicos e/ou extraesofágicos, associados ou não a lesões teciduais. Convém destacar três aspectos importantes na definição apresentada:

- Admite-se a participação de componentes do refluxo duodenogástrico na fisiopatogenia da afecção. Em função disso, propõe-se o termo refluxo de conteúdo gastroduodenal, e não apenas de conteúdo gástrico.

- Admite-se a existência de sintomas esofágicos e extraesofágicos.

- Destaca-se que os sintomas podem ou não ser acompanhados por lesões teciduais esofágicas diagnosticadas pelo exame endoscópico. 


\section{PREVALÊNCIA}

A DRGE é afecção de grande importância médico-social pela elevada e crescente incidência e por determinar sintomas de intensidade variável, que se manifestam por tempo prolongado, podendo prejudicar consideravelmente a qualidade de vida do paciente. Tem prevalência estimada de $20 \%$ na população adulta dos Estados Unidos ${ }^{3}$, com taxas similares na Europa, e de 6 a 17\% da população idosa ${ }^{4}$.

Em revisão sistemática de literatura abrangendo 15 publicações, publicada em 2005, destaca-se que a prevalência da doença varia de 10 a 20\% no mundo ocidental e é ao redor de 5\% na Ásia, considerando-se indicador sugestivo da DRGE a presença de pirose e/ou regurgitação ácida, ao menos 1 vez/semana. São apontados os seguintes fatores de risco para desenvolvimento da doença: história familiar, obesidade, idade, tabagismo, entre outros. Refere-se aumento da incidência com aumento da idade até a $5^{\text {a }}$ década de vida; a partir de então, não se nota aumento expressivo ${ }^{5}$. Essa revisão foi ampliada em 2014, com inclusão de 15 novas publicações, agregando dados do Oriente Médio, Austrália e América do Sul. O único país da América do Sul citado foi a Argentina, com prevalência de sintomas de DRGE de $23 \%{ }^{6}$.

No Brasil, estudo populacional empreendido em 22 metrópoles, entrevistando-se amostra populacional de 13.959 indivíduos $^{7}$, observou que 4,6\% das pessoas avaliadas apresentavam pirose $1 \mathrm{vez} /$ semana e que $7,3 \%$ apresentavam tal queixa 2 ou mais vezes/semana. Em função desses dados, estima-se que a prevalência da DRGE, em nosso meio, seja ao redor de $12 \%$.

Há poucas publicações específicas sobre a prevalência da doença em diferentes faixas etárias; uma delas, na população japonesa, refere prevalência de $10,3 \%$ na população geral, sendo: $18,4 \%$ entre 60 e 69 anos, $17,6 \%$ entre 70 e 79 anos e $21,1 \%$ nas pessoas acima de 80 anos $^{8}$. Em contraste, estudo brasileiro avaliando 3.050 adultos indicou que os sintomas de refluxo não variam significativamente em função da idade? .

Convém salientar que as manifestações atípicas do refluxo são mais comuns em idosos e que a DRGE nessa faixa etária está associada a alterações endoscópicas, histológicas e motoras mais intensas ${ }^{10}$. Como os estudos sobre prevalência da afecção baseiam-se na presença dos chamados sintomas típicos, é provável que subestimem a real ocorrência da DRGE na população idosa.

\section{FISIOPATOLOGIA}

Muitos fatores fisiopatológicos estão envolvidos na DRGE. A principal barreira anatômica contra o refluxo gastresofágico (RGE) é a junção 
esofagogástrica (JEG), região anatomicamente complexa cuja integridade depende da competência do esfíncter inferior do esôfago (EIE) e da compressão extrínseca deste pela crura diafragmática $(\mathrm{CD})^{11}$. O EIE é um segmento da musculatura lisa de 3 a $4 \mathrm{~cm}$ de extensão, tonicamente contraído durante o repouso, com pressão de repouso de 10 a $30 \mathrm{mmHg}$ e certa capacidade de reserva funcional; ou seja, com pressão superior aos 5 a $10 \mathrm{mmHg}$, que seria suficiente para prevenir $\mathrm{RGE}^{12}$. O EIE é circundado pela $\mathrm{CD}$ que promove compressão extrínseca ao EIE, contribuindo para a pressão de relaxamento e incrementando a pressão do EIE nos momentos de aumento súbito da pressão abdominal, como inspiração profunda, tosse e espirro ${ }^{13}$.

Um dos principais mecanismos envolvidos na fisiopatologia da DRGE é a ocorrência de relaxamentos transitórios do EIE (RTEIE), os quais são definidos como breves períodos de relaxamento do EIE espontâneos e não relacionados com a deglutição ${ }^{14}$. Constituem mecanismo fisiológico que permite esvaziamento do excesso de gás do estômago e podem ocorrer tanto em pacientes com DRGE quanto em voluntários sadios. A distensão gástrica causada por ar ou alimento desencadeia reflexo vagal que resulta em RTEIE, atuando como mecanismo protetor que previne o acúmulo de ar intragástrico, impedindo que o excesso de gás vá para o duodeno.

Dent et al. descreveram pela primeira vez os RTEIE em 10 voluntários sadios na década de $1980^{15}$. Desde então, RTEIE vêm sendo descritos como principal mecanismo fisiopatológico nos pacientes com DRGE. Quando foram inicialmente identificados, foi aventada a hipótese de que pacientes com DRGE teriam maior taxa de RTEIE. No entanto, vários estudos relataram similaridade entre taxa de RTEIE em pacientes com DRGE e indivíduos saudáveis ${ }^{16-19}$. Posteriormente, foi demonstrado que o refluxo ácido ocorre com maior frequência durante os RTEIE em pacientes com DRGE do que nos indivíduos saudáveis ${ }^{20}$.

A presença de hérnia hiatal $(\mathrm{HH})$ também está envolvida na fisiopatologia da DRGE, no entanto, com relevância controversa. HH é definida como a migração cranial de elementos da cavidade abdominal, mais comumente o estômago, através do hiato diafragmático ${ }^{21}$. Dados epidemiológicos e fisiológicos confirmam a importância da $\mathrm{HH}$ em pacientes com esofagite mais grave, estenoses pépticas e esôfago de Barrett ${ }^{13}$.

A patogênese da hérnia hiatal por deslizamento já foi tema de vários estu$\operatorname{dos}^{21-24}$. Diversas teorias foram propostas, sendo mais aceita a que relaciona a sequência de eventos determinada por RTEIE resultando em exposição ácida no esôfago distal. A consequente redução do $\mathrm{pH}$ esofágico leva a seu encurtamento, em virtude da contração da musculatura longitudinal induzida pelo ácido e à fibrose causada pela esofagite. Esses fatores, associados à perda da elasticidade das estruturas de apoio, como a membrana frenoesofágica, ine- 
rente à idade cronológica do paciente, e consequente alargamento da abertura diafragmática causada pela porção herniada, contribuem para maior exposição ácida, perpetuando o ciclo vicioso.

Outros fatores relacionados à $\mathrm{HH}$ por deslizamento são o aumento da pressão intra-abdominal e a obesidade. A pressão abdominal parece ser fator de risco isolado independente da obesidade, haja vista a alta prevalência (até 80\%) de $\mathrm{HH}$ em atletas de força ${ }^{25}$. A associação de obesidade com hérnia hiatal por deslizamento e DRGE foi extensivamente estudada ${ }^{26-28}$. Pandolfino et al. analisaram a relação entre o índice de massa corpórea (IMC) e a circunferência abdominal com a morfologia do segmento pressórico na JEG, por meio de manometria de alta resolução, demostrando que a obesidade está associada à ruptura da JEG e ao surgimento de $\mathrm{HH}^{29}$.

A hérnia hiatal está associada à redução da pressão e do comprimento da JEG funcional, em consequência da separação axial entre EIE e CD e da perda do segmento abdominal do esfíncter ${ }^{11}$, o que pode desencadear consequências clínicas e fisiológicas, como redução da pressão do EIE e da amplitude peristáltica no esôfago distal e comprometimento da depuração ácida do esôfago. Portanto, pode desencadear DRGE e suas complicações, em especial, esôfago de Barrett ${ }^{22,30}$.

Outro fator envolvido na patogênese da DRGE é a disfunção peristáltica do corpo do esôfago, que tem influência direta na capacidade de depuração do refluxato. É sabido que a motilidade esofágica ineficaz (MEI) correlaciona-se diretamente com a intensidade da DRGE e com a presença das suas complicações ${ }^{31}$. No entanto, ainda não se sabe se é causa ou consequência da DRGE ${ }^{32}$.

Há outras condições envolvidas na fisiopatologia da DRGE; dentre elas, destaca-se o fato de o conteúdo ácido da porção mais proximal da região da cárdia "escapar" do efeito neutralizante do alimento e manter-se acidificado durante o período pós-prandial. Esse efeito, chamado "acid pocket"33,34, pode se estender por até $1 \mathrm{a} 2 \mathrm{~cm}$ no esôfago distal e desencadear sintomas ${ }^{35}$.

Em idosos, há várias condições que podem favorecer o aparecimento da DRGE e das suas complicações; dentre elas, destacam-se: maior ocorrência de distúrbios motores do esôfago, secreção salivar reduzida, menor pressão do EIE, enfraquecimento diafragmático com maior incidência de hérnia hiatal, presença de comorbidades como doença de Parkinson e diabetes e uso de medicações para outros problemas médicos, que podem afetar a função esofágica ${ }^{36}$.

\section{MANIFESTAÇÕES CLÍNICAS E DIAGNÓSTICO}

O primeiro passo para o diagnóstico adequado da DRGE é o conhecimento do conceito atual da afecção e das suas várias formas de apresentação clínica. As manifestações clínicas consideradas típicas da DRGE são pirose e regurgitação. 
Apesar dessas queixas sugerirem a presença da afecção, vale salientar que outras doenças também podem desencadear tais sintomas. Contudo, quando tais queixas coexistem, a possibilidade do paciente ser portador da DRGE é superior a 90\%.

Outras manifestações clínicas como dor torácica não coronariana, sensação de globus faríngeo, manifestações extraesofágicas respiratórias (fundamentalmente, tosse e asma brônquica) e otorrinolaringológicas (basicamente, disfonia e pigarro), também podem ser decorrentes de RGE. Vale lembrar que a ausência de manifestações típicas do refluxo não exclui o diagnóstico da DRGE. Pacientes com manifestações atípicas frequentemente não apresentam sintomas típicos do refluxo ${ }^{37}$.

Em adultos jovens, são cabíveis dois tipos de abordagem inicial em pacientes com suspeita de DRGE: tratamento empírico (teste terapêutico) e tratamento baseado na confirmação diagnóstica por exames subsidiários. Na decisão sobre a abordagem a ser adotada, é importante considerar a idade e a presença ou não de manifestações de alarme. Recomenda-se confirmação diagnóstica em pacientes com idade superior a 40 anos na presença de manifestações de alarme como disfagia, odinofagia, anemia, hemorragia digestiva e emagrecimento, nos indivíduos com história familiar de câncer e naqueles com queixas de náuseas e vômitos e/ou sintomas de grande intensidade ou de ocorrência noturna ${ }^{38-40}$. Em idosos, não se recomenda iniciar o tratamento empírico sem avaliação endoscópica do trato digestivo alto, para excluir afecções associadas.

Ainda é polêmica a questão dos sintomas do refluxo em idosos. Locke et al. refere que a prevalência de pirose declina com o avançar da idade; porém, a de regurgitação, não ${ }^{4}$. Contudo, em revisão sistemática recente envolvendo 14 estudos sobre DRGE em idosos, aponta-se que não ocorre aumento de prevalência das queixas de refluxo com a idade, mas, apesar disso, há maior ocorrência de esofagite erosiva à endoscopia e piora do padrão de refluxo à monitoração prolongada ${ }^{41}$. Em estudo retrospectivo envolvendo análise de sintomas e achados endoscópicos em mais de 12.000 pacientes, salienta-se que a ocorrência de esofagite erosiva mais intensa (graus C e D) é maior em idosos. Em contrapartida, a ocorrência de queixas típicas tende a diminuir ${ }^{42}$.

Collen et al. ${ }^{43}$ descrevem presença de esofagite erosiva em $81 \%$ de pacientes com DRGE e idade maior que 60 anos e em $47 \%$ na população mais jovem. Salientam também que a ocorrência de esôfago de Barrett também é maior em idosos: $25 \%$ vs. $15 \%$.

Em função disso, salienta-se que há desproporção ainda maior que a observada em indivíduos jovens, entre as intensidades dos sintomas típicos e da DRGE. Ou seja, idosos podem não ter tantos sintomas típicos, mas apresentam maior ocorrência de complicações da doença e de outras queixas, como disfagia, dor torácica e sangramento digestivo relacionado a DRGE. 


\section{INDICAÇÕES E LIMITAÇÕES DOS PRINCIPAIS MÉTODOS DIAGNÓSTICOS}

\section{Endoscopia digestiva alta (EDA)}

Avalia a presença e o grau de esofagite (é considerado o melhor método para tal diagnóstico). Vale lembrar que existe esofagite endoscópica em cerca de 50\% dos pacientes com DRGE com predomínio de manifestações típicas (pirose e regurgitação) e em apenas 15 a 20\% daqueles com predomínio de manifestações atípicas. Portanto, a ausência de esofagite ao estudo endoscópico não exclui o diagnóstico da DRGE ${ }^{44}$.

A EDA também caracteriza a presença de complicações da DRGE (esôfago de Barrett, estenose e ulcerações esofágicas) e evidencia afecções associadas (hérnia hiatal, úlceras pépticas gastroduodenais, neoplasias etc.).

Como limitações, a EDA não avalia o RGE, mas somente suas eventuais consequências.

No consenso de Lyon, publicado recentemente ${ }^{45}$, considera-se como bons indicativos da presença de DRGE as esofagites erosivas graus C e D e o esôfago de Barrett longo; as formas mais leves de esofagite erosiva e o Barrett curto seriam indicativos menos consistentes da DRGE. A forma não erosiva da doença requer monitoração prolongada do refluxo para sua real caracterização.

\section{Monitoração prolongada do RGE}

Avalia a presença e a intensidade do refluxo; caracteriza o padrão de refluxo (ortostático, supino ou combinado) e relaciona a queixa clínica com o refluxo. A modalidade mais difundida de monitoração prolongada do refluxo - a pHmetria - avalia apenas a modalidade mais comum de refluxo, ou seja, o refluxo líquido ácido. A impedâncio-pHmetria avalia todas modalidades de refluxo (ácido, não ácido, líquido e gasoso), porém, com custo mais elevado e disponibilidade mais restrita.

Está indicada para as seguintes situações:

- Identificação dos portadores da DRGE sem esofagite.

- Caracterização do padrão de refluxo.

- Estudo da participação do refluxo ácido manifestações atípicas do refluxo.

- Estudo da recidiva dos sintomas no pós-operatório.

- Avaliação da eficácia do tratamento clínico; nessa situação, como destacado a seguir, a impedâncio-pHmetria esofágica apresenta nítida superioridade em relação à pHmetria convencional. 
Sua limitações é que não avalia a presença de esofagite e de suas complicações.

A impedâncio-pHmetria apresenta vantagens expressivas em relação à pHmetria nas seguintes situações:

- Estudo das queixas clínicas refratárias ao tratamento farmacológico do refluxo. Nessa situação, o exame dever ser realizado na vigência da droga em uso, para saber se os sintomas são decorrentes de refluxo ácido não adequadamente bloqueado, de refluxo não ácido ou se não são relacionados com o refluxo.

- Estudo das manifestações atípicas de refluxo, em especial, na tosse crônica.

- Estudo das eructações, para caracterização dos subtipos: gástricas e supragástricas.

A EDA e a monitoração prolongada do refluxo são os dois métodos diretamente relacionados com o diagnóstico da DRGE. O primeiro identifica o subgrupo da doença que cursa com esofagite, e o segundo caracteriza o grupo com RGE patológico sem esofagite. A EDA propicia ainda a realização de coleta de material de biópsia para estudo histológico. Tal procedimento é de fundamental importância no estudo das complicações da doença. A seguir, são destacadas as indicações e as limitações de outros métodos diagnósticos que, apesar de não se prestarem para o diagnóstico da DRGE em si, têm utilidade na prática clínica.

\section{Estudo radiológico contrastado do esôfago}

\section{Indicações}

- Propicia boa avaliação morfológica do órgão (forma do esôfago e características do trânsito da substância de contraste).

- Pode evidenciar complicações da DRGE (estenoses e ulcerações) e condições favorecedoras do refluxo (hérnia hiatal e ângulo esofagogástrico anormal).

\section{Limitações}

- Não identifica esofagite (apenas eventuais complicações desta).

- Não caracteriza adequadamente o RGE.

Manometria esofágica (convencional ou de alta resolução)

Avalia o tônus pressórico dos esfíncteres do esôfago e a atividade motora do corpo esofágico ${ }^{46}$. 


\section{Indicações}

Fornece bom parâmetro preditivo de evolução da doença. Casos com hipotonia muito acentuada do EIE frequentemente necessitam de tratamento medicamentoso continuado para controle adequado dos sintomas. Nesses casos, cogita-se mais precocemente a opção do tratamento cirúrgico do refluxo.

- Diagnóstico de distúrbios motores específicos do esôfago que podem alterar a orientação terapêutica, como acalasia, aperistalse e hipocontratilidades muito acentuadas.

- Fornece bom parâmetro referencial da atividade motora no pré-operatório. Na presença de complicação disfágica no pós-operatório de fundoplicatura, a avaliação manométrica do esôfago tem mais chance de auxiliar no esclarecimento da causa da disfagia nos pacientes submetidos a manometria pré-operatória.

- Identificação da localização dos esfíncteres do esôfago para posicionamento adequado dos sensores de monitoração prolongada do refluxo. Para esse fim, não é necessário estudo manométrico completo do esôfago; realiza-se estudo sumário, apenas para identificação dos esfíncteres.

\section{Limitações}

- Não avalia a real capacidade que o esôfago tem de transportar as substâncias ingeridas. Há alterações manométricas que comprometem bastante a função esofágica de transporte, como acalasia e acometimento importante do esôfago por colagenose. Por outro lado, há alterações que não comprometem substancialmente o transporte do bolus ${ }^{47}$.

Em idosos, a suspeita de DRGE deve ser inicialmente investigada por meio de EDA. O estudo radiológico contrastado nessa faixa etária pode caracterizar detalhes importantes sobre a morfologia do órgão, evidências indiretas de eventuais distúrbios motores e de condições associadas, como hérnias hiatais e divertículos. O estudo radiológico dinâmico, com registro em vídeo, é bastante superior ao estudo radiológico convencional, em especial em idosos, por viabilizar a análise das três fases da deglutição: oral, faríngea e esofágica.

A investigação diagnóstica mais invasiva, por manometria e monitoração prolongada do refluxo, deve ser restrita aos casos nos quais eventuais achados desses exames justifiquem mudanças na abordagem terapêutica. 


\section{TRATAMENTO CLÍNICO}

Os tratamentos clínico e cirúrgico têm maior risco em idosos do que em jovens10; portanto, merecem cuidados especiais.

O tratamento clínico visa ao alívio dos sintomas, à cicatrização das lesões e à prevenção de recidivas e complicações. Do ponto de vista prático, objetiva-se reduzir o potencial agressivo do conteúdo gástrico, minimizando a agressão representada pelo ácido clorídrico do suco gástrico ${ }^{38}$.

Pode-se classificar a abordagem terapêutica em medidas comportamentais e farmacológicas, que devem ser implementadas simultaneamente.

\section{Medidas comportamentais}

As medidas comportamentais são apresentadas na Tabela 1.

Tabela 1 Medidas comportamentais no tratamento da DRGE'

Elevação da cabeceira da cama $(15 \mathrm{~cm})$

Moderação na ingestão dos seguintes alimentos, na dependência da correlação com os sintomas: gordurosos, citricos, café, bebidas alcoólicas, bebidas gasosas, menta, hortelã, produtos de tomate, chocolate

Cuidados especiais para medicamentos potencialmente "de risco": anticolinérgicos, teofilina, antidepressivos tricíclicos, bloqueadores de canais de cálcio, agonistas beta-adrenégicos, alendronato

Evitar deitar-se nas 2 horas após as refeições

Evitar refeições copiosas

Redução ou cessação do fumo

Reduzir o peso corporal (emagrecimento)

Apesar de não haver estudos suportados por critérios de medicina baseada em evidências em favor da utilização das medidas comportamentais, a experiência clínica tem sugerido fortemente a sua utilização (recomendação nível C).

\section{Medidas farmacológicas}

Os inibidores de bomba de prótons (IBP) aliviam os sintomas do refluxo e cicatrizam a esofagite mais rapidamente que os antagonistas $\mathrm{H}_{2}\left(\mathrm{AH}_{2}\right)^{48,49}$. As doses plenas diárias utilizadas são apresentadas na Tabela 2. 
Tabela 2 Inibidores da bomba protônica e suas doses plenas diárias ${ }^{1}$

\begin{tabular}{ll} 
IBP & Dose plena diária \\
\hline Omeprazol & $40 \mathrm{mg}$ \\
\hline Lansoprazol & $30 \mathrm{mg}$ \\
\hline Pantoprazol & $40 \mathrm{mg}$ \\
\hline Rabeprazol & $20 \mathrm{mg}$ \\
\hline Esomeprazol & $40 \mathrm{mg}$ \\
\hline Dexlansoprazol & $60 \mathrm{mg}$
\end{tabular}

Contudo, apesar da eficiência dos IBP, como já destacado, existem casos que não têm boa resposta à droga. Há tendência em tratar os pacientes com diagnóstico de esofagite com IBP, em dose plena, por 6 a 12 semanas. Alguns autores $^{50}$ propõem administração de dose dobrada para as esofagites de maior gravidade (graus III e IV de Savary-Miller ou C e D de Los Angeles).

Estudo baseado em revisão sistemática de literatura ${ }^{51}$, avaliando a eficiência de IBP e $\mathrm{AH}_{2}$ em adultos com sintomas típicos da DRGE sem esofagite ao estudo endoscópico, conclui que "os agentes antissecretores são eficientes no controle da queixa clínica desses pacientes e que os IBP apresentam resultados melhores que os $\mathrm{AH}_{2}$ ".

Um aspecto importante a ser considerado é que os IBP controlam adequadamente os sintomas ácido-dependentes da DRGE, mas, não têm efeito adequado no controle de sintomas decorrentes da presença física do refluxo (independentes da sua acidez). Vela et al. ${ }^{52}$, avaliando por meio de impedâncio-pHmetria esofágica a eficiência do omeprazol no controle do RGE, observaram que o uso do fármaco não reduzia significantemente o número de episódios de refluxo, mas sim alterava sua acidez; continua havendo o mesmo número de refluxos, contudo, os refluxos tornavam-se não ácidos. Portanto, com o uso da droga, espera-se melhora clínica dos sintomas ácido-dependentes, explicando-se a manutenção da queixa de sintomas não ácido-dependentes.

Shay et al. ${ }^{53}$, estudando, por meio de impedâncio-pHmetria, pacientes que permanecem sintomáticos apesar do uso de IBP, observaram que $28 \%$ deles apresentavam sintomas relacionados com refluxo ácido (detectável por pHmetria convencional). Contudo, $40 \%$ deles apresentavam sintomas relacionados com refluxo não ácido (detectável pela impedâncio-pHmetria). Os autores descrevem que, nos $32 \%$ restantes, os sintomas não foram relacionados com refluxo (ácido e não ácido).

Apesar dessa observação não ser consensual, alguns estudos sugerem que idosos necessitam de maior supressão ácida para resolução da esofagite erosiva que adultos jovens ${ }^{54}$. $\mathrm{O}$ uso de IBP em idosos merece algumas considerações: 
- Apesar de haver redução da depuração plasmática de IBP com o avançar da idade, não se preconiza a redução da dose empregada em idosos, mesmo na presença de algum grau de disfunção hepática e renal ${ }^{55}$.

- Omeprazol e lansoprazol são metabolizados sob influência do citocromo P450 e podem afetar o metabolismo de outras drogas ${ }^{56}$.

- Recomenda-se cautela em pacientes idosos em uso de vários medicamentos com vias similares de metabolização, em especial varfarina, fenitoína, diazepam e carbamazepina. Um dos antiagregantes plaquetários mais usados - o clopidogrel - é metabolizado pelo mesmo citocromo que alguns IBP. Em função disso, pode haver redução da sua ação em pacientes em uso desses tipos de IBP ${ }^{57}$. Contudo, essa questão é bastante controversa, e diretrizes recentemente publicadas ${ }^{58}$ minimizam a importância clínica da interferência do uso de certos IBP na ação do clopidogrel.

- Pode haver consequências nutricionais, metabólicas e infecciosas decorrentes do uso crônico de IPB. Pode ocorrer redução da absorção de vitamina B12, porém, sem impacto clínico relevante ${ }^{59}$. Pode haver redução da absorção de cálcio, com consequente redução da densidade óssea e eventual maior risco de fraturas ${ }^{60}$, porém, a relevância clínica dessa observação é bastante contestada. Há maior ocorrência de diarreia por Clostridium difficile $^{61}$; portanto, em pacientes em uso crônico de IBP que apresentem diarreia, essa possibilidade deve ser lembrada e investigada.

- O nível sérico de magnésio pode decair com o uso crônico de IBP ${ }^{62}$. Apesar da monitoração dos níveis plasmáticos de magnésio ser sugerida, questiona-se a relevância clínica dessa conduta.

Há algumas publicações relacionando o uso crônico de IBP com o risco de câncer de estômago e cólon ${ }^{63-67}$. Recentemente, foram publicados dados de uma coorte populacional incluíndo 800.000 adultos suecos que utilizaram IBP por pelo menos 6 meses, apontando aumento de risco de câncer gástrico da ordem de 3 vezes ${ }^{65}$. Outro estudo recentemente publicado, avaliando pacientes após tratamento do Helicobacter pylori, também descreve certa elevação do risco de câncer gástrico. No entanto, tal estudo foi de caráter retrospectivo e não considerou outros fatores de risco para câncer gástrico, como história familiar, idade, tabagismo, obesidade e alcoolismo. Desta forma, não há conclusões definitivas sobre o aumento do risco de câncer relacionados ao uso crônico dos IBP. Mais estudos são necessários para melhor entendimento dessa eventual relação.

Estudos de associação entre o uso de IBP e demência têm levantado resultados conflitantes ${ }^{68,69}$. Sabe-se que o uso de IBP tem aumentado expressivamente, sobretudo na população geriátrica. Nessa população, a doença de Alzheimer é 
um distúrbio neurológico comum e pode ocorrer com frequência de até $10 \%{ }^{70}$. No entanto, estudos de base populacionais publicados recentemente não demonstraram associação entre o uso de IBP e demência ou Alzheimer ${ }^{71,72}$. Novos estudos randomizados controlados e pesquisas básicas que avaliem o mecanismo envolvido nessa eventual relação são necessários.

Convém salientar que o uso dos IBP tem se tornado abusivo e, por vezes, injustificado. Pasina et al. descrevem que $40 \%$ dos idosos admitidos para algum tipo de tratamento hospitalar estavam em uso de IBP, porém, com indicação não justificada em $66 \%$ deles $^{73}$. Sem dúvidas, os IBP, assim como qualquer outra medicação, não devem ser utilizados sem indicações objetivas e adequadamente justificadas.

Apesar dos IBP serem os antissecretores mais eficientes no tratamento clínico da DRGE, alguns pacientes podem ser convenientemente controlados com antissecretores menos potentes - os bloqueadores $\mathrm{H}_{2}$. Salienta-se, contudo, que, em idosos, devem ser evitadas doses elevadas dessas drogas em função da maior ocorrência de efeitos colaterais, sobretudo nos casos com algum grau de insuficiência hepática ou renal ${ }^{74}$. $\mathrm{O}$ uso de alcalinos também deve ser criterioso em idosos, pelo potencial risco de sobrecarga de sais, constipação, diarreia e eventuais interferências na absorção de outras drogas ${ }^{10}$.

As drogas procinéticas, úteis nos casos com síndrome dispéptica associa à DRGE, também merecem cautela em idosos. A metoclopramida pode causar efeitos colaterais, como tremores musculares, espasmos, agitação, ansiedade, insônia e confusão mental em cerca de um terço dos pacientes ${ }^{75}$.

\section{TRATAMENTO CIRÚRGICO}

Evidentemente, a idade do paciente é um fator muito importante a ser considerado na decisão da opção terapêutica a ser empregada. Idosos em boa condição física, com baixo risco cirúrgico, podem ser operados. Há algumas revisões comparando sintomas pré e pós-operatórios e ocorrência de complicações em pacientes em duas faixas etárias: superior a 70 e inferior a 60 anos. Cowgill et al. descrevem que a pontuação dos sintomas, pré e pós-operatórios é menor nos idosos e que os índices de bons resultados e complicações são semelhantes nos dois grupos estudados. Salienta-se, contudo, que essa observação é válida para idosos muito bem selecionados em relação ao risco operatório e a presença de comorbidades ${ }^{76}$. Pizza et al. descrevem ocorrência maior de sintomas atípicos e de distúrbios motores nos indivíduos idosos ${ }^{77}$.

Os candidatos ao tratamento cirúrgico devem ser meticulosamente avaliados, em especial os idosos. Além da avaliação clínica adequada do risco cirúrgico de eventuais comorbidades, devem ser consideradas a atividade motora 
do esôfago e, em alguns casos, o padrão de refluxo. Além do estudo endoscópico, recomendam-se:

- Estudo radiológico contrastado do esôfago para avaliação morfológica deste.

- Estudo manométrico do esôfago (convencional ou de alta resolução) para diagnóstico de eventuais distúrbios motores que contraindiquem o tratamento cirúrgico e para servir de referencial pré-operatório nos casos que eventualmente evoluam com algum grau de disfagia.

- Monitoração prolongada do refluxo (pHmetria ou impedâncio-pHmetria) nos casos em que o diagnóstico da DRGE não é embasado com segurança pelo estudo endoscópico. No recente consenso de Lyon, salienta-se que apenas as esofagites erosivas de maior intensidade (graus C e D na Classificação de Los Angeles) e o esôfago de Barrett longo representam bons marcadores endoscópicos da DRGE. Portanto, recomenda-se que os pacientes com formas menos intensas de esofagite erosiva (A e B) e esôfago de Barrett curto sejam submetidos a monitoração prolongada do refluxo. Os casos com comprovação de refluxo patológico pela monitoração prolongada apresentam melhores resultados do tratamento cirúrgico que os casos nos quais o refluxo patológico é suspeitado, mas não comprovado ${ }^{45}$.

A ocorrência de grandes herniações hiatais pode representar um desafio adicional nos idosos. Apenas os casos bastante sintomáticos ou com complicações decorrentes da hérnia devem ser cogitados para o tratamento cirúrgico. $\mathrm{O}$ tratamento cirúrgico dessas grandes hérnias tem taxas de complicações e recidiva expressivas; por isso, a opção cirúrgica deve ser reservada a casos muito bem selecionados.

Por muito tempo, preconizou-se que o tratamento cirúrgico da DRGE estaria indicado nos casos de "intratabilidade clínica" e nas formas complicadas da doença. Com o significativo aumento da eficiência das drogas usadas no tratamento clínico da afecção, pode-se dizer que, atualmente, é menos frequente a observação de "intratabilidade clínica", devendo os pacientes em tal situação ser meticulosamente estudados para conferir a real participação do RGE no desencadeamento dos sintomas. O que se observa na prática é que a maioria dos pacientes tem boa resposta às medidas clínicas usualmente empregadas. Entretanto, parte significativa dos enfermos (cerca de 50\%) necessita manter um tratamento clínico prolongado (comportamental e/ou medicamentoso) para se manter assintomática ${ }^{78}$.

Atualmente, o ponto crucial da indicação do tratamento cirúrgico é a tolerância ao tratamento clínico prolongado. Alguns pacientes preferem manter restrições comportamentais e, frequentemente, o uso de medicamentos a ser 
operados; outros preferem o contrário. Cabe ao médico apresentar ao paciente, com imparcialidade, as opções terapêuticas, ponderando vantagens e desvantagens. A decisão sobre a modalidade de tratamento a ser utilizada deve ser compartilhada com o paciente e seus familiares.

\section{TRATAMENTO ENDOSCÓPICO}

Várias modalidades de tratamento endoscópico da DRGE (plicatura endoscópica, aplicação de radiofrequência e injeção de polímeros na transição esofagogástrica) têm sido desenvolvidas. Contudo, até o presente momento, a maior parte dos estudos avalia os resultados de curto prazo dessas intervenções. Tais estudos têm evidenciado melhora da queixa de pirose, mas não normalização do refluxo à monitoração prolongada nem cicatrização da esofagite endoscópica. Algumas complicações, incluindo óbitos, têm sido relatadas. Até o momento, não há evidências suficientes para recomendar a utilização de tais modalidades de tratamento na prática clínica assistencial. Os estudos relativos ao tema devem continuar restritos a centros de pesquisa e investigação, até que se produzam resultados mais consistentes.

Estudo baseado em revisão sistemática de literatura ${ }^{79}$ avaliando o tratamento endoscópico da DRGE conclui que: "apesar de haver resultados favoráveis, não há estudos bem controlados suficientes que justifiquem a aplicação clínica do tratamento endoscópico do refluxo". No entanto, revisão recentemente publicada avaliando 4 técnicas endoscópicas com acompanhamento de até 48 meses demonstrou melhora da qualidade de vida e sintomas, com perfil de segurança favorável. Nesta revisão, entre 41 e $77 \%$ dos pacientes permaneceram livres de medicamentos antirrefluxo ${ }^{80}$.

\section{CONSIDERAÇÕES FINAIS}

Com base na revisão ora apresentada, pode-se salientar que:

- Há poucas publicações sobre a prevalência da DRGE em idosos; as existentes sugerem prevalência maior em idosos que na população geral.

- Há dados conflitantes sobre a ocorrência de queixas típicas. Algumas publicações sugerem que a ocorrência de pirose se reduza com a idade e que a de regurgitação se mantenha. No entanto, outras apontam que não há diferenças expressivas em quanto à prevalência das queixas típicas em relação à faixa etária.

- Entretanto, parece claro que os idosos apresentam maior ocorrência de fatores favorecedores da DRGE e das suas complicações; dentre esses, desta- 
cam-se: maior incidência de distúrbios motores do esôfago, secreção salivar reduzida, menor pressão do EIE, enfraquecimento diafragmático com maior incidência de hérnia hiatal, presença de comorbidades como doença de Parkinson e diabetes, e uso de medicações para outros problemas médicos que podem afetar a função esofágica.

- Há desproporção ainda maior que a observada em indivíduos jovens entre as intensidades dos sintomas típicos e da DRGE. Ou seja, idosos podem não ter tantos sintomas típicos, mas apresentam maior ocorrência de complicações da doença e de outras queixas, como disfagia, dor torácica e sangramento digestivo relacionado a DRGE.

- Apesar dos testes terapêuticos serem válidos na população não idosa, em idosos, não se recomenda iniciar o tratamento empírico sem avaliação endoscópica do trato digestivo alto, para excluir afecções associadas.

- Em idosos, a suspeita de DRGE deve ser inicialmente investigada por meio de EDA. O estudo radiológico contrastado nessa faixa etária pode caracterizar detalhes importantes sobre a morfologia do órgão, evidências indiretas de eventuais distúrbios motores e de condições associadas, como hérnias hiatais e divertículos. O estudo radiológico dinâmico, com registro em vídeo, é bastante superior ao estudo radiológico convencional, em especial em idosos, por viabilizar a análise das três fases da deglutição: oral, faríngea e esofágica.

- A investigação diagnóstica mais invasiva, por manometria e monitoração prolongada do refluxo, deve ser restrita aos casos nos quais eventuais achados desses exames justifiquem mudanças na abordagem terapêutica.

- Os tratamentos clínico e cirúrgico têm maior risco em idosos que em jovens; portanto, merecem cuidados especiais.

- Apesar dos IBP serem os antissecretores mais eficientes no tratamento clínico da DRGE, alguns pacientes podem ser convenientemente controlados com antissecretores menos potentes - os bloqueadores $\mathrm{H}_{2}$. Salienta-se, contudo, que, em idosos, devem ser evitadas doses elevadas dessas drogas em função da maior ocorrência de efeitos colaterais, sobretudo nos casos com algum grau de insuficiência hepática ou renal. $\mathrm{O}$ uso de alcalinos também deve ser criterioso em idosos, pelo potencial risco de sobrecarga de sais, constipação, diarreia e eventuais interferências na absorção de outras drogas.

- Idosos em boas condições clínicas podem ser submetidos ao tratamento cirúrgico, porém, com critérios de indicação meticulosamente avaliados e ponderados em relação ao risco-benefício.

- Várias modalidades de tratamento endoscópico da DRGE têm sido desenvolvidas. Contudo, até o presente momento, não há evidências suficientes que autorizem a utilização do tratamento endoscópico do refluxo na 


\section{prática clínica assistencial. Os estudos relativos ao tema devem continuar restritos a centros de pesquisa e investigação, até que se produzam resulta- dos mais consistentes.}

\section{REFERÊNCIAS BIBLIOGRÁFICAS}

1. Moraes-Filho JPP, Cecconello I, Gama-Rodrigues J, De Paula Castro L, Henry MA, Meneghelli UG, et al. Brazilian consensus on gastroesophageal reflux disease: proposals for assessment, classification, and management. Am J Gastroenterol. 2002;97(2):241-8.

2. Moraes-Filho JPP, Navarro-Rodriguez T, Barbuti R, Eisig J, Chinzon D, Bernardo W, et al. Guidelines for the diagnosis and management of gastroesophageal reflux disease: an evidence-based consensus. Arq Gastroenterol. 2010;47(1):99-115.

3. Sonnenberg A, El-Serag HB. Clinical epidemiology and natural history of gastroesophageal reflux disease. Yale J Biol Med. 1999;72(2-3):81-92.

4. Locke GR 3rd, Talley NJ, Fett SL, Zinsmeister AR, Melton LJ 3rd. Prevalence and clinical spectrum of gastroesophageal reflux: a population-based study in Olmsted County, Minnesota. Gastroenterology. 1997;112(5):1448-56.

5. Dent J, El-Serag HB, Wallander M-A, Johansson S. Epidemiology of gastro-oesophageal reflux disease: a systematic review. Gut. 2005;54(5):710-7.

6. El-Serag HB, Sweet S, Winchester CC, Dent J. Update on the epidemiology of gastro-oesophageal reflux disease: a systematic review. Gut. 2014;63(6):871-80.

7. Moraes-Filho JPP, Chinzon D, Eisig JN, Hashimoto CL, Zaterka S. Prevalence of heartburn and gastroesophageal reflux disease in the urban Brazilian population. Arq Gastroenterol. 2005; 42(2):122-7.

8. Okimoto E, Ishimura N, Morito Y, Mikami H, Shimura S, Uno G, et al. Prevalence of gastroesophageal reflux disease in children, adults, and elderly in the same community. J Gastroenterol Hepatol. 2015;30(7):1140-6.

9. De Oliveira Latorre MDRD, Da Silva AM, Chinzon D, Eisig JN, Dias-Bastos TRP. Epidemiology of upper gastrointestinal symptoms in Brazil (EpiGastro): a population-based study according to sex and age group. World J Gastroenterol. 2014;20(46):17388-98.

10. Achem SR, DeVault KR. Gastroesophageal reflux disease and the elderly. Gastroenterol Clin North Am. 2014;43(1):147-60.

11. Kahrilas PJ. The role of hiatus hernia in GERD. Yale J Biol Med. 1999;72(2-3):101-11.

12. Dodds WJ, Dent J, Hogan WJ, Helm JF, Hauser R, Patel GK, et al. Mechanisms of gastroesophageal reflux in patients with reflux esophagitis. N Engl J Med. 1982;307(25):1547-52.

13. Richter JE. Gastrooesophageal reflux disease. Best Pract Res Clin Gastroenterol. 2007;21(4):609-31.

14. Kim HI, Hong SJ, Han JP, Seo JY, Hwang KH, Maeng HJ, et al. Specific movement of esophagus during transient lower esophageal sphincter relaxation in gastroesophageal reflux disease. J Neurogastroenterol Motil. 2013;19(3):332-7.

15. Dent J, Dodds WJ, Friedman RH, Sekiguchi T, Hogan WJ, Arndorfer RC, et al. Mechanism of gastroesophageal reflux in recumbent asymptomatic human subjects. J Clin Invest. 1980;65(2):256-67.

16. Massey BT, Simuncak C, Lecapitaine-Dana NJ, Pudur S. Transient lower esophageal sphincter relaxations do not result from passive opening of the cardia by gastric distention. Gastroenterology. 2006;130(1):89-95.

17. Iwakiri K, Hayashi Y, Kotoyori M, Tanaka Y, Kawakami A, Sakamoto C, et al. Transient lower esophageal sphincter relaxations (TLESRs) are the major mechanism of gastroesophageal reflux but are not the cause of reflux disease. Dig Dis Sci. 2005;50(6):1072-7. 
18. Trudgill NJ, Riley SA. Transient lower esophageal sphincter relaxations are no more frequent in patients with gastroesophageal reflux disease than in asymptomatic volunteers. Am J Gastroenterol. 2001;96(9 SUPPL.):2569-74.

19. Grossi L, Ciccaglione AF, Travaglini N, Marzio L. Transient lower esophageal sphincter relaxations and gastroesophageal reflux episodes in healthy subjects and GERD patients during 24 hours. Dig Dis Sci. 2001;46(4):815-21.

20. Bredenoord AJ, Weusten BLAM, Timmer R, Smout AJPM. Gastro-oesophageal reflux of liquids and gas during transient lower oesophageal sphincter relaxations. Neurogastroenterol Motil. 2006;18(10):888-93.

21. Hyun JJ, Bak Y-T. Clinical significance of hiatal hernia. Gut Liver. 2011;5(3):267-77.

22. Gordon C, Kang JY, Neild PJ, Maxwell JD. The role of the hiatus hernia in gastro-oesophageal reflux disease. Aliment Pharmacol Ther. 2004;20(7):719-32.

23. Mittal RK. Hiatal hernia: myth or reality? Am J Med. 1997;103(5A):33S-39S.

24. Christensen J, Miftakhov R. Hiatus hernia: a review of evidence for its origin in esophageal longitudinal muscle dysfunction. Am J Med. 2000;108 Suppl:3S-7S.

25. Smith AB, Dickerman RD, McGuire CS, East JW, McConathy WJ, Pearson HF. Pressure-overload-induced sliding hiatal hernia in power athletes. J Clin Gastroenterol. 1999;28(4):352-4.

26. Wilson LJ, Ma W, Hirschowitz BI. Association of obesity with hiatal hernia and esophagitis. Am J Gastroenterol. 1999;94(10):2840-4.

27. Barak N, Ehrenpreis ED, Harrison JR, Sitrin MD. Gastro-oesophageal reflux disease in obesity: pathophysiological and therapeutic considerations. Obes Rev. 2002;3(1):9-15.

28. Stene-Larsen G, Weberg R, Frøyshov Larsen I, Bjørtuft O, Hoel B, Berstad A. Relationship of overweight to hiatus hernia and reflux oesophagitis. Scand J Gastroenterol. 1988;23(4):427-32.

29. Pandolfino JE, El-Serag HB, Zhang Q, Shah N, Ghosh SK, Kahrilas PJ. Obesity: a challenge to esophagogastric junction integrity. Gastroenterology. 2006;130(3):639-49.

30. Kahrilas PJ, Kim HC, Pandolfino JE. Approaches to the diagnosis and grading of hiatal hernia. Best Pract Res Clin Gastroenterol. 2008;22(4):601-16.

31. Kahrilas PJ, Dodds WJ, Hogan WJ, Kern M, Arndorfer RC, Reece A. Esophageal peristaltic dysfunction in peptic esophagitis. Gastroenterology. 1986;91:897-904.

32. Daum C, Sweis R, Kaufman E, Fuellemann A, Anggiansah A, Fried M, et al. Failure to respond to physiologic challenge characterizes esophageal motility in erosive gastro-esophageal reflux disease. Neurogastroenterol Motil. 2011;23(6):517-e200.

33. Fletcher J, Wirz A, Young J, Vallance R, McColl KE. Unbuffered highly acidic gastric juice exists at the gastroesophageal junction after a meal. Gastroenterology. 2001;121(4):775-83.

34. Lee YY, McColl KE. Pathophysiology of gastroesophageal reflux disease. Best Pr Res Clin Gastroenterol. 2013;27(3):339-51.

35. Lee YY, Whiting JGH, Robertson EV, Derakhshan MH, Smith D, McColl KEL. Measuring movement and location of the gastroesophageal junction: research and clinical implications. Scand J Gastroenterol. 2013;48(4):401-11.

36. Bashashati M, Sarosiek I, McCallum RW. Epidemiology and mechanisms of gastroesophageal reflux disease in the elderly: a perspective. Ann N Y Acad Sci. 2016;1380(1):230-4.

37. Nasi A, Filho JP, Zilberstein B, Cecconello I, Gama-Rodrigues JJ, Pinotti HW. Gastroesophageal reflux disease: clinical, endoscopic, and intraluminal esophageal $\mathrm{pH}$ monitoring evaluation. Dis Esophagus. 2001;14(1):41-9.

38. DeVault KR, Castell DO. Updated guidelines for the diagnosis and treatment of gastroesophageal reflux disease. Am J Gastroenterol. 2005;100(1):190-200.

39. Dent J, Brun J, Fendrick A, Fennerty M, Janssens J, Kahrilas P, et al. An evidence-based appraisal of reflux disease management - the Genval Workshop Report. Gut. 1999;44 Suppl 2:S1-16.

40. Moss SF, Arnold R, Tytgat GN, Spechler SJ, Delle-Fave G, Rosin D, et al. Consensus Statement for Management of Gastroesophageal Reflux Disease: result of workshop meeting at Yale Univer- 
sity School of Medicine, Department of Surgery, November 16 and 17, 1997. J Clin Gastroenterol. 1998;27(1):6-12.

41. Becher A, Dent J. Systematic review: ageing and gastro-oesophageal reflux disease symptoms, oesophageal function and reflux oesophagitis. Aliment Pharmacol Ther. 2011;33(4):442-54.

42. Johnson DA, Fennerty MB. Heartburn Severity Underestimates Erosive Esophagitis Severity in Elderly Patients with Gastroesophageal Reflux Disease. Gastroenterology. 2004;126(3):660-4.

43. Collen MJ, Abdulian JD, Chen YK. Gastroesophageal reflux disease in the elderly: more severe disease that requires aggressive therapy. Am J Gastroenterol 1995;90(7):1053-7.

44. Streets CG, DeMeester TR. Ambulatory 24-hour esophageal pH monitoring: why, when, and what to do. J Clin Gastroenterol. 2003;37(1):14-22.

45. Gyawali CP, Kahrilas PJ, Savarino E, Zerbib F, Mion F, Smout AJPM, et al. Modern diagnosis of GERD: the Lyon Consensus. Gut. 2018;67(7):1351-62.

46. Pandolfino JE, Kahrilas PJ, American Gastroenterological Association. AGA technical review on the clinical use of esophageal manometry. Gastroenterology. 2005;128(1):209-24.

47. Tutuian R, Castell DO. Clarification of the esophageal function defect in patients with manometric ineffective esophageal motility: studies using combined impedance-manometry. Clin Gastroenterol Hepatol. 2004;2(3):230-6.

48. Chiba N, De Gara CJ, Wilkinson JM, Hunt RH. Speed of healing and symptom relief in grade II to IV gastroesophageal reflux disease: a meta-analysis. Gastroenterology. 1997;112(6):1798-810.

49. Katelaris P, Holloway R, Talley N, Gotley D, Williams S, Dent J. Gastro-oesophageal reflux disease in adults: guidelines for clinicians. J Gastroenterol Hepatol. 2002;17(8):825-33.

50. Orlando RC. Why is the high grade inhibition of gastric acid secretion afforded by proton pump inhibitors often required for healing of reflux esophagitis? An epithelial perspective. Am J Gastroenterol. 1996;91(9):1692-6.

51. Sigterman KE, van Pinxteren B, Bonis PA, Lau J, Numans ME. Short-term treatment with proton pump inhibitors, $\mathrm{H} 2$-receptor antagonists and prokinetics for gastro-oesophageal reflux disease-like symptoms and endoscopy negative reflux disease. Cochrane Database Syst Rev. 2013;(5):CD002095.

52. Vela MF, Camacho-Lobato L, Srinivasan R, Tutuian R, Katz PO, Castell DO. Simultaneous intraesophageal impedance and $\mathrm{pH}$ measurement of acid and nonacid gastroesophageal reflux: effect of omeprazole. Gastroenterology. 2001;120(7):1599-606.

53. Shay S, Tutuian R, Sifrim D, Vela M, Wise J, Balaji N, et al. Twenty-four hour ambulatory simultaneous impedance and $\mathrm{pH}$ monitoring: a multicenter report of normal values from 60 healthy volunteers. Am J Gastroenterol. 2004;99(6):1037-43.

54. Garnett WR, Garabedian-Ruffalo SM. Identification, diagnosis, and treatment of acid-related diseases in the elderly: Implications for long-term care. Pharmacotherapy. 1997;17(5):938-58.

55. McTavish D, Buckley MM, Heel RC. Omeprazole. An updated review of its pharmacology and therapeutic use in acid-related disorders. Drugs. 1991;42(1):138-70.

56. Andersson T. Pharmacokinetics, metabolism and interactions of acid pump inhibitors. Focus on omeprazole, lansoprazole and pantoprazole. Clin Pharmacokinet. 1996;31(1):9-28.

57. Laine L, Hennekens C. Proton pump inhibitor and clopidogrel interaction: fact or fiction? Am J Gastroenterol. 2010;105(1):34-41.

58. Katz PO, Gerson LB, Vela MF. Guidelines for the diagnosis and management of gastroesophageal reflux disease. Am J Gastroenterol. 2013;108(3):308-28.

59. Saltzman JR, Kemp JA, Golner BB, Pedrosa MC, Dallal GE, Russell RM. Effect of hypochlorhydria due to omeprazole treatment or atrophic gastritis on protein-bound vitamin B12 absorption. J Am Coll Nutr. 1994;13(6):584-91.

60. Yang Y-X, Lewis JD, Epstein S, Metz DC. Long-term proton pump inhibitor therapy and risk of hip fracture. JAMA. 2006;296(24):2947-53.

61. DeVault KR, Talley NJ. Insights into the future of gastric acid suppression. Nat Rev Gastroenterol Hepatol. 2009;6(9):524-32. 
62. Cundy T, Dissanayake A. Severe hypomagnesaemia in long-term users of proton-pump inhibitors. Clin Endocrinol (Oxf). 2008;69(2):338-41.

63. Eusebi LH, Rabitti S, Artesiani ML, Gelli D, Montagnani M, Zagari RM, et al. Proton pump inhibitors: risks of long-term use. J Gastroenterol Hepatol. 2017;32(7):1295-302.

64. Brusselaers N, Engstrand L, Lagergren J. Maintenance proton pump inhibition therapy and risk of oesophageal cancer. Cancer Epidemiol. 2018;53:172-7.

65. Brusselaers N, Wahlin K, Engstrand L, Lagergren J. Maintenance therapy with proton pump inhibitors and risk of gastric cancer: a nationwide population-based cohort study in Sweden. BMJ Open. 2017;7(10):e017739.

66. Ahn JS, Eom C-S, Jeon CY, Park SM. Acid suppressive drugs and gastric cancer: a meta-analysis of observational studies. World J Gastroenterol. 2013;19(16):2560-8.

67. Cheung KS, Chan EW, Wong AYS, Chen L, Wong ICK, Leung WK. Long-term proton pump inhibitors and risk of gastric cancer development after treatment for Helicobacter pylori: a population-based study. Gut. 2018;67(1):28-35.

68. Wod M, Hallas J, Andersen K, Garcia Rodriguez LA, Christensen K, Gaist D. Lack of association between proton pump inhibitor use and cognitive decline. Clin Gastroenterol Hepatol. 2018;16(5):681-9.

69. Haenisch B, von Holt K, Wiese B, Prokein J, Lange C, Ernst A, et al. Risk of dementia in elderly patients with the use of proton pump inhibitors. Eur Arch Psychiatry Clin Neurosci. 2015;265(5):41928.

70. Ide K, Matsuoka N, Kawakami K. Is the use of proton-pump inhibitors a risk factor for Alzheimer's disease? Molecular mechanisms and clinical implications. Curr Med Chem. 2018;25(18):2166-74.

71. Hwang IC, Chang J, Park SM. A nationwide population-based cohort study of dementia risk among acid suppressant users. Am J Geriatr Psychiatry. 2018;pii:S1064-7481(18)30362-2.

72. Imfeld P, Bodmer M, Jick SS, Meier CR. Proton pump inhibitor use and risk of developing Alzheimer's disease or vascular dementia: a case-control analysis. Drug Saf. 2018;doi: 10.1007/s40264018-0704-9. [Epub ahead of print]

73. Pasina L, Nobili A, Tettamanti M, Salerno F, Corrao S, Marengoni A, et al. Prevalence and appropriateness of drug prescriptions for peptic ulcer and gastro-esophageal reflux disease in a cohort of hospitalized elderly. Eur J Intern Med. 2011;22(2):205-10.

74. Lipsy RJ, Fennerty B, Fagan TC. Clinical review of histamine 2 receptor antagonists. Arch Intern Med. 1990;150(4):745-51.

75. Verlinden M. Review article: a role for gastrointestinal prokinetic agents in the treatment of reflux oesophagitis? Aliment Pharmacol Ther. 1989;3(2):113-31.

76. Cowgill SM, Arnaoutakis D, Villadolid D, Al-Saadi S, Arnaoutakis D, Molloy DL, et al. Results after laparoscopic fundoplication: does age matter? Am Surg. 2006;72(9):774-8.

77. Pizza F, Rossetti G, Limongelli P, Del Genio G, Maffettone V, Napolitano V, et al. Influence of age on outcome of total laparoscopic fundoplication for gastroesophageal reflux disease. World J Gastroenterol. 2007;13(5):740-7.

78. Guidelines for surgical treatment of gastroesophageal reflux disease (GERD). Society of American Gastrointestinal Endoscopic Surgeons (SAGES). Surg Endosc 1998;12(2):186-8.

79. Johnson DA. Endoscopic therapy for gastroesophageal reflux disease: a systematic review. Minerva Gastroenterol Dietol. 2004;50(3):239-51.

80. Reyes Genere J, Wang KK. Endoscopic techniques for treating gastroesophageal reflux. Curr Opin Gastroenterol. 2018;34(5):288-94. 